Marly Apareci da Elias Cosendey 1 Jorge Antônio Zepeda Bermudez 2 André Luis de Almeida dos Reis 2 Hayne Feli pe da Silva 2 Maria Auxiliadora Oliveira 2 Vera Lúcia Luiza 3

\section{Assistência farmacêutica na atenção básica de saúde: a experiência de três estados brasileiros}

\author{
Provision of essential medicines \\ within basic health care: the experience \\ of three Brazilian States
}

\footnotetext{
1 Serviço de Farmácia, Hospital Universitário Clementino Fraga Filho, Universidade Federal do Rio de Janeiro. Av. Brigadeiro Trompowsky $\mathrm{s} / \mathrm{n}$, Il ha do Fundão, Rio de Janeiro, RJ 21941-590, Brasil. marlycosendey@openlink. com.br

2 Núcleo de Assistência Farmacêutica, Escola Nacional de Saúde Pública, Fundação Oswaldo Cruz. Rua Leopoldo Bulhões 1480, Rio de Janeiro, RJ 21045-900, Brasil. bermudez@ensp.fiocruz.br 3 Hospital Evandro Chagas, Fundação Oswaldo Cruz. Av. Brasil 4365, Manguinhos, Rio de Janeiro, RJ 21045-900, Brasil.
}

Abstract In the context of recent changes in the Brazilian drug supply system at the national level, mainly the deactivation of CEME (a government agency under the Ministry of Heal th that dealt with drug supply policy) and the increasing decentralization of activities under the Unified National Health System (SUS), three Brazilian States - Paraná, São Paulo, and Minas Gerais - have established programs for the distribution of essential drugs for primary health care. A nationwide program for the procurement and supply of 32 drugs for primary health care (the socalled "Basic Pharmacy Program") is currently being implemented by the Ministry of Health, to be decentralized subsequently. This article discusses the establi shment of State-level drug policies for primary health care through the analysis of the three pioneering programs encompassing drug selection, procurement, storage, distribution, and use.

Key words Essential Drugs; Drug Policy; Drug Assistance

Resumo No contexto das mudanças ocorri das no sistema de fornecimento de medicamentos em nível nacional no Brasil, com destaque para a desativação da Central de Medicamentos (CEME), e da crescente descentralização das ações de saúde no âmbi to do Si stema Único de Saúde (SUS), três estados brasilei ros - Paraná, São Paulo e Minas Gerais - elaboraram, dentro da política de assi stência farmacêutica, programas envolvendo a distribui ção de medi camentos essenciais para a atenção primária de saúde. O Ministério da Saú de está atualmente implementando um programa nacional de aquisi ção e distribui ção de um el enco de 32 medicamentos para a atenção primária ("Programa Farmácia Bási ca") que deverá ter suas ati vi dades posteriormente descentralizadas. A discussão aqui proposta tem por objetivo fornecer subsídios para a organização e/ou estruturação da assi stência farmacêutica estadual no que concerne à atenção primária de saúde, por meio da análi se destes três programas pionei ros, envolvendo as ati vidades de sel eção, aquisição, estocagem, distribuição e uso de medicamentos.

Palavras-chave Medicamentos Essenciais; Política de Medicamentos; Assistência Farmacêutica 
Introdução

Uma pesquisa realizada pela Organização Mundial da Saúde - OMS (WHO, 1988) com 104 países em desenvolvimento mostra que em 24 destes países, menos de $30 \%$ da população têm acesso regular aos medicamentos essenciais; em 33 países, entre 30\% e 60\% da população e, apenas nos 47 países restantes, mais de $60 \%$. Observa-se, portanto, que a necessidade da maioria da população em relação ao medicamento não é satisfeita. As diferenças na disponibilidade de medicamentos entre os países estão relacionadas a questões políticas referentes aos medicamentos essenciais, assim como às suas situações financeiras. O acesso a esses medicamentos é limitado em países pobres devido, principalmente, a duas razões: os preços destes produtos são altos para a média de rendimentos de suas populações e os medicamentos necessários ao tratamento das principais doenças não se encontram disponíveis.

O Brasil está entre os cinco maiores consumidores de medicamentos do mundo com vendas anuais em torno de $\mathrm{R} \$ 11,1$ bilhões (SINDUSFARM, 1998). Entretanto, cerca de $60 \%$ da produção de medicamentos do país beneficiam apenas $23 \%$ da população. A análise do mercado brasileiro mostra que os produtos registrados e comercializados deixam muito a desejar no que se refere à qual idade, com $20 \%$ dos produtos não correspondendo às especificações da própria indústria farmacêutica, nas vendas extra-hospitalares (Bermudez \& Possas, 1995). Ainda assim, os preços dos medicamentos no Brasil chegam a ser 20 vezes maiores que os preços internacionais (Abbas \& Bermudez, 1993; Bermudez, 1994).

No final do ano de 1992 a crise de medicamentos no Brasil agravou-se devido a problemas de aumento dos preços de venda destes, decorrentes do fim do controle de preços por parte do governo. Estudos realizados durante o período de abril de 1992 e abril de 1993 mostram que, frente a uma inflação estimada em $1.608 \%$, os preços de al guns medicamentos aumentaram em 2.600\% (Bermudez \& Possas, 1995), na tentativa de recuperar a margem de lucro perdida durante o período com controle governamental. Isto dificultou ainda mais o acesso da população aos medicamentos.

Em 1997, o governo brasileiro lançou um programa de distribuição de medicamentos essenciais denominado Programa Farmácia Básica (PFB) com o objetivo de possibilitar o acesso da população a estes medicamentos na atenção primária de saúde. Este programa está estruturado em duas etapas. Na primeira eta- pa, que compreende o período de dezembro/97 a julho/98, estão sendo distribuídos os medicamentos que compõem a relação da Farmácia Básica para os municípios com população menor que 21.000 habitantes. A partir de agosto de 1998, o governo pretende efetuar apenas o repasse da verba referente ao gasto com medicamentos, que deverá ser administrada pelas secretarias estaduais de saúde (MS, 1997a, 1997b, 1997c).

A discussão aqui proposta tem por objetivo fornecer subsídios para a organização e/ ou estruturação da assistência farmacêutica estadual no que concerne à atenção primária de saúde, mediante análise realizada em fevereiro de 1998, de três programas pioneiros de fornecimento de medicamentos essenciais para este nível de atenção, desenvolvidos pelos estados do Paraná, São Paulo e Minas Gerais.

Na primeira parte será abordada de forma breve a história da Central de Medicamentos (CEME), a fim de facilitar o entendimento da iniciativa desses três estados e inserir as suas experiências no contexto da política nacional de medicamentos. Na segunda parte serão descritos e discutidos os programas de medicamentos adotados pelos estados analisados. As informações foram obtidas nos documentos oficiais e de visitas, quando foram entrevistados os gestores dos programas. Utilizou-se, para tanto, um roteiro padronizado de entrevista com o objetivo de apreender os aspectos relacionados aos componentes dos programas e padronizar a forma de abordagem das principais questões, como a seleção de medicamentos, definição da clientela, sistemas de aquisição e distribuição de medicamentos e gerenciamento dos programas.

CEME, RENAME e Farmácia Básica: concepção, falência e iniciativas estaduais

A CEME foi criada em 25 de junho de 1971 pelo Decreto 68.806, tendo como objetivos principais a promoção e a organização das atividades de assistência farmacêutica aos estratos populacionais de reduzido poder aquisitivo, o incremento à pesquisa científica e tecnológica no campo químico-farmacêutico e o incentivo à instalação de fábricas de matérias-primas e de laboratórios pilotos. Entre os seus objetivos específicos, ressalta-se a identificação de indicadores, como a incidência de doenças por região e por faixa de renda, o levantamento da capacidade de produção dos laboratórios farmacêuticos, a racionalização das categorias 
básicas de medicamentos por especialidades, a organização de eventos científicos, o planejamento e a coordenação de mecanismos de distribuição e venda de medicamentos em todo o território nacional (Bermudez, 1995).

A CEM E passou por várias fases desde sua criação. Por ser um órgão que estava ligado diretamente à Presidência da República, estaria sujeita às influências das várias correntes ideológicas e dos interesses políticos que predominavam na época. Por isso, desviava-se dos seus objetivos iniciais, na medida em que o contexto político se alterava.

Em 1975 foi instituída a Relação Nacional de Medicamentos Essenciais (RENAME) pela Portaria no 223 do Ministério da Previdência e Assistência Social, com a proposta de ser periodicamente revisada (Bermudez, 1995).

A RENAME era um instrumento estratégico da Política Nacional de Medicamentos. Técnicos da CEME, após diversos estudos, chegaram a listar 305 substâncias farmacêuticas (fármacos básicos) que possibilitariam atender $99 \%$ das necessidades médicas da população, racionalizando os critérios e procedimentos de compra (Pereira, 1995).

Após 15 anos da última revisão publicada no país, a RENAME foi finalmente atualizada em 1998, seguindo diretrizes recomendadas pela OMS, tendo por base a revisão feita em 1993 pela CEME, mas não divulgada, além de revisão da literatura mundial recente. Nesta nova edição, conferiu-se ênfase à avaliação de segurança e eficácia, além de se considerar a respeito da disponibilidade dos produtos no mercado interno. A nova lista contém 303 princípios ativos em 545 apresentações destinadas a atender as principais nosologias prevalentes no Brasil (MS, 1998).

Embora a RENAME seja uma lista de medicamentos prioritários, o seu fornecimento por parte do governo para as unidades de saúde foi deficiente durante um grande período da existência da CEME. "Ainda assim, a RENAME simboliza um esforço na direção de uma assi stência farmacêutica mais eficaz e de mel hor qualidade" (Pepe \& Veras, 1995:19).

Em 1987, entrou em operacionalização a Farmácia Básica, que foi uma proposta governamental para racionalizar o fornecimento de medicamentos para a atenção primária de saúde (Bermudez, 1992). Foi idealizada como um módulo-padrão de suprimento de medicamentos selecionados da RENAME, que permitiam o tratamento das doenças mais comuns da população brasileira, especialmente aquelas voltadas para o nível ambulatorial. Os módulospadrão foram planejados para atender as ne- cessidades de três mil pessoas por um período de seis meses, constando de 48 medicamentos. Posteriormente, foram acrescentados à Farmácia Básica medicamentos de uso contínuo, totalizando 60 medicamentos considerados de maior demanda na rede pública de saúde (Medici et al., 1991; Bermudez, 1995).

Em 1987, ano em que o programa foi implantado, apenas 3.370 dos 4.600 municípios brasileiros foram beneficiados com a Farmácia Básica. As descontinuidades nos fornecimentos nos anos posteriores impediram que este programa tivesse um desempenho mais satisfatório. Em 1989 o programa contemplou 50 milhões de pessoas (Medici et al., 1991), por intermédio de mais de 19.200 farmácias básicas implantadas (Castelo et al., 1991).

Para Bermudez (1995:137) “o módulo deFarmácia Básica podeser identificado, em grande parte, como um instrumento de favorecimento do clientel ismo político que contribui u para a distorção da CEME de suas finalidades iniciais e para o quadro de esvaziamento político que caracterizou as consecutivas mudanças em sua vinculação institucional".

Embora na concepção da Farmácia Básica tenham sido consideradas as diversidades regionais, a sua implantação por um módulo-padrão em nível nacional não contemplou estas diversidades. O mesmo módulo-padrão era fornecido para todas as regiões do Brasil que apresentam perfis epidemiológicos totalmente diferenciados. Este fato teve como conseqüência a falta de alguns medicamentos e o excesso de outros, o que incorria em desperdício. A falta de planejamento na produção e distribuição dos medicamentos do programa fazia com que as unidades de saúde recebessem muitos medicamentos com a data de validade próxima ao vencimento, o que promovia grandes perdas, pois todas as unidades encontravam-se abarrotadas destes produtos, impossibilitando inclusive seu remanejamento.

Em 1988, com a promulgação da Nova Constituição Federal do Brasil, foi conferido ao Estado o papel de assegurar a saúde de forma universal, integral e equânime, incluindo a assistência farmacêutica. Apesar disto, no período compreendido entre 1991 e 1992, a CEME reduziu a $20 \%$ seus níveis históricos de cobertura da demanda de medicamentos para o SUS (Bermudez, 1995).

Para Pereira (1995:95) “a partir de 1990, com o advento do governo Collor, os problemas de indefini ção dos objetivos da CEME se agravaram. A CEME passou por uma fase deagitação e mudanças caóticas. Sua estrutura administrativa foi desmontada usando-se como pretexto a 
modernização, sem na verdade ter nada deconcreto para colocar no lugar, denotando uma típica ação de sabotagem, que por sorte, veio à tona com os escândal os do governo Collor".

Esta série de escândalos culminou com a desativação da CEME e o realinhamento estratégico das competências, planos, programas e projetos a cargo da mesma (Bermudez et al., 1997). Desta forma, as suas atividades foram pulverizadas em diferentes órgãos do Ministério da Saúde, como Secretaria de Vigilância Sanitária, Secretaria de Políticas de Saúde, Secretaria Executiva e Secretaria de Projetos Especiais de Saúde, esta última posteriormente extinta.

Neste contexto e devido à inoperância do sistema CEME, os governos de três estados brasileiros - Paraná, São Paulo e Minas Gerais - elaboraram no âmbito da política de assistência farmacêutica, programas que incluem a distribuição de medicamentos essenciais para a atenção primária. Estes programas apresentam como objetivo comum garantir o tratamento eficaz das patologias mais freqüentes em cada estado, expandindo o acesso e a resolubilidade da rede no atendimento das necessidades individuais e coletivas da população de baixa renda, integralizando a distribuição e dispensação de medicamentos às ações de saúde em nível básico. Entretanto, os programas de cada estado apresentam particularidades que serão apresentadas a seguir.

Estratégias com um objetivo comum: expandir o acesso aos medicamentos

Estado do Paraná: uma proposta

pioneira na esfera estadual

O Paraná foi o primeiro estado brasileiro a propor uma política de medicamentos destinada aos cuidados primários de saúde. O governo do estado conferiu prioridade, dentre os projetos estratégi cos a serem desenvolvidos na área de saúde, à organização da Assistência Farmacêutica na rede de serviços públicos (GAF/SESPR, 1995). Para tanto, foram organizadas discussões para a estruturação de uma política estadual de medicamentos que tiveram como propostas as seguintes operações: diagnóstico das necessidades de medicamentos no atendimento básico ambulatorial e hospitalar; estabelecimento da Lista de Medicamentos Básicos (LMB); avaliação de medicamentos de al to custo; organização do sistema estadual de produção de medicamentos; vigilância sanitária e qualidade de medicamentos.

O programa de medicamentos proposto para o atendimento dos cuidados primários de saúde denominou-se Programa Farmácia Básica (PFB-PR), seguindo o modelo proposto pela CEME em 1987, com algumas adequações e aprimoramentos. A elaboração da relação dos medicamentos que fariam parte do PFB-PR considerou como critérios de inclusão os seguintes: os medicamentos deveriam constar da RENAME; serem eficazes, seguros e disponíveis para aquisição ao menor custo possível. Desta forma, foi elaborada uma lista contendo 32 princípios ativos em 40 apresentações que poderiam ser adquiridos por meio da CEM E, produção no CEMEPAR (Centro de Medicamentos do Paraná), em laboratórios públicos estaduais conveniados, laboratórios oficiais de outros estados ou de laboratórios privados e, neste caso, pelo processo licitatório. A quantificação dos medicamentos foi feita para grupos de 3.000 habitantes, utilizando-se como base para os cálculos, as informações fornecidas pelos municípios e a lista de medicamentos da Farmácia Básica da CEME de 1987, estabelecendo-se assim um módulo-padrão para um período de três meses. Após preparada a relação de medicamentos do PFB-PR, ela foi enviada a todos os município para avaliação das quantidades e dos medicamentos selecionados, sendo o momento adequado para propostas de alterações da lista.

Como contrapartida os municípios incumbiram-se de organizar a assistência à saúde, estruturando sua rede dentro do preconizado pelo SUS; garantir que os fóruns específicos possibilitem debate e controle social, sendo democráticos e representativos, incluindo gestores, prestadores e usuários; assegurar investimentos e financiamentos crescentes para o setor de saúde; estruturar área física adequada à guarda de medicamentos e imunobiológicos; racionalizar a prescrição médica e a dispensação farmacêutica; estabelecer condutas terapêuticas para o tratamento das patologias predominantes e estimular a padronização de medicamentos em consonância com estas condutas; implementar mecanismos de controle sobre a utilização dos medicamentos de programas específicos e de uso contínuo; disponibilizar e capacitar recursos humanos em saúde, necessários para garantir uma assistência farmacêutica de qualidade; apresentar trimestralmente à Regional de Saúde da área, Mapa Resumo de Consumo da Farmácia Básica devidamente preenchido e assinado por autoridade sanitária municipal, assim como demonstrativo qualitativo e quantitativo das aquisições de medicamentos feitas pelo município; intercambiar informações técnicas e experiências relacionadas à seleção, aquisição, distribuição e utiliza- 
ção de medicamentos; criar instrumentos que colaborem no processo de conscientização e esclarecimento da população sobre o uso correto dos medicamentos; responsabilizar-se, frente à Secretaria Estadual de Saúde, pela distribuição exclusiva à rede SUS dos medicamentos enviados pelo PFB-PR, garantindo prescrição e dispensação adequadas.

O programa foi implantado em abril de 1995 e os critérios utilizados para a seleção dos municípios que recebem o PFB-PR foram dados populacionais atualizados e o Índice de Salubridade (IS). O cálculo deste índice contemplou indicadores de saúde, sociais e de serviços (Strozzi, 1997). Desta forma, cada município recebeu uma pontuação referente ao índice de salubridade. Quanto maior o IS, melhor a situação do município em relação aos indicadores avaliados. De acordo com este índice, os municípios foram ordenados e subdivididos em quatro grupos por ordem de prioridade: prioridade 1 (menor IS), recebem $100 \%$ das cotas previstas, segundo base populacional; prioridade 2 , recebem $75 \%$; prioridade 3 , recebem $50 \%$ e prioridade 4 , recebem $25 \%$. Mediante racionalização dos recursos disponíveis utilizando-se dos critérios descritos anteriormente, todos os municípios do estado recebem medicamentos do PFB-PR, em quantidades variáveis e dependentes do IS. Em fevereiro de 1998, o PFB-PR atendia a uma população de cerca de 3.909.000 habitantes, que corresponde a aproximadamente $43 \%$ da população do estado.

O gasto mensal com o programa é de cerca de $R \$ 912.000$ (U\$796.500), que equivale a um custo individual de cerca de $R \$ 0,23 / \mathrm{hab} /$ mês $(\mathrm{U} \$ 0,20)$.

O processo de distribuição de medicamentos é centralizado e começa com a emissão de guias de remessa e separação por destinatário feita pelo CEM EPAR. Os medicamentos são encaminhados às Regionais de Saúde, acompanhados das guias de remessa com as cotas destinadas a cada município que integra a Regional. As Regionais encarregam-se da separação e repasse dos medicamentos às Secretarias Municipais de Saúde que, por sua vez incumbem-se da distribuição às unidades de saúde, mediante critérios estabel ecidos pelas instâncias gestoras locais. O controle da distribuição dos medicamentos é realizado nos três níveis (central, regional e local) segundo guias de remessa e inventários trimestrais de estoque e dispensação de medicamentos e tem por objetivo evitar perdas, faltas ou excessos de medicamentos. Entretanto, é de ressaltar que, ao seguir o modelo CEME, cada município recebe o número de módulos-padrão, que é composto por quantidades fixas sempre dos mesmos medicamentos, independente do consumo dos produtos.

O controle de estoque no nível central (CEMEPAR) é informatizado, o que permite uma programação mais eficiente da distribuição, além de facilitar o controle dos prazos de validade e possibilitar a manutenção de estoques em quantidades adequadas às necessidades. No depósito do CEM EPAR são recolhidas amostras dos produtos e enviadas aos laboratórios credenciados para realização das análises físico-químicas e microbiológicas.

Com o objetivo de incentivar a prescrição racional de medicamentos e assegurar uma assistência farmacêutica de qualidade, foram elaborados e distribuídos materiais educativos que contêm informações sobre farmacologia clínica e terapêutica (guia farmacoterapêutico), além de outros materiais informativos para a equipe de saúde e usuários e o desenvolvimento de cursos, com ênfase nos medicamentos, para os profissionais de saúde envolvidos.

Estado de São Paulo: dose certa - Programa de Assistência Farmacêutica

No estado de São Paulo, o Programa de Assistência Farmacêutica (PAF) passou por um período de planejamento que durou cerca de oito meses. Nesta fase foi feito o diagnóstico das necessidades de medicamentos em nível de atenção primária e selecionado o elenco de medicamentos essenciais que fariam parte do PAF, de acordo com os seguintes critérios: demanda histórica de consumo de medicamentos, fornecida pelos municípios; adequação à Sétima Lista de Medicamentos Essenciais da OMS; capacidade de produção do medicamento pela FURP (Fundação para o Remédio Popular), que possibilita a garantia da continuidade do fornecimento com medicamentos de qualidade. Utilizando-se o critério de demanda histórica, foram selecionados inicialmente 75 medicamentos, que foram reduzidos a 38 produtos, pela utilização dos dois outros critérios citados anteriormente. Sendo a lista composta por 32 princípios ativos em 40 apresentações que, segundo a secretária-executiva da Comissão de Medicamentos Básicos, Harue Ohashi, cobre entre 70 e $80 \%$ das patologias registradas nas Unidades Básicas de Saúde (UBSs). A quantificação dos medicamentos foi feita para grupos de 1.000 habitantes com base na demanda histórica com o objetivo de atender 100\% das necessi dades (CSI/SESSP, 1996; DPA/ CSI/SESSP, 1996; GTPA/CSI/ SESSP, 1996; FURP, $S / d)$. 
Em contrapartida ao recebimento dos medicamentos do PAF, os municípios comprometem-se em reordenar e melhorar a assistência à saúde; organizar farmácia local obedecendo às boas práticas de estocagem; fornecer insulina, cujo abastecimento pelo Ministério da Saúde, através do programa de diabetes, não vem atendendo às necessidades dos serviços; estruturar a área física em condições adequadas à guarda de medicamentos; racionalizar a prescrição e a dispensação de medicamentos; estabelecer protocolos de tratamentos para as patologias predominantes; implementar mecanismos de controle sobre o consumo de medicamentos do programa; apresentar mensalmente à Diretoria Regional de Saúde (DIR) mapa de consumo dos medicamentos do PAF, preenchido e assinado por autoridade sanitária local; disponibilizar e capacitar recursos humanos para o PAF; distribuir os medicamentos somente na rede do SUS a pacientes cadastrados nas UBSs. O não cumprimento destas obrigações incorre em suspensão provisória do fornecimento de medicamentos, podendo ocorrer o descredenciamento do município.

Após a fase de planejamento, iniciou-se em agosto de 1995 a realização de um projeto piloto nos municípios da região de Sorocaba, uma das mais pobres do estado, conhecida como ramal da fome. O projeto piloto incluiu, além do fornecimento de medicamentos, um seminário de implantação com o objetivo de promover a integração dos profissionais de saúde de cada unidade que receberia os medicamentos do PAF; capacitar os gestores municipais e regionais para a sua implantação e sensibilizálos para o compromisso, viabilização e construção em parceria, respeitando as realidades regionais e locais. Os participantes destes seminários eram os médicos e farmacêuticos das UBSs, prefeitos, coordenadores e secretários de saúde.

O critério utilizado para inclusão dos municípios no PAF foi de base populacional ea ordem de inclusão foi a disponibilidade e a mobilização de cada regional para a realização dos seminários de implantação. A implantação do PAF na Coordenadoria de Saúde do Interior (CSI ) ocorreu em três fases (CSI/ SESSP, 1998). Na primeira fase, foram incluídos 491 municípios com população de até 30.000 habitantes, atendendo a 5.382 .367 pessoas; na segunda fase, foram incluídos 90 municípios com população até 120.000 habitantes, atendendo mais 5.473.069 pessoas; e na terceira fase, foram incluídos 14 municípios com população até 250.000 habitantes, atendendo a mais 2.501.369 pessoas. Em fevereiro de 1998, dos 606 municí- pios da CSI, 595 são atendidos pelo PAF, cobrindo uma população de 13.356.805 habitantes, que representa $74,7 \%$ de sua população e cerca de $45 \%$ da população total do estado. Encontra-se em fase de estudo a ampliação do PAF para o restante do estado.

Em outubro de 1995, foi implantado nos municípios participantes do programa o Sistema de Informação do Programa de Assistência Farmacêutica (SISPAF), que permitiu conhecer o consumo real e a disponibilidade dos medicamentos do programa em cada município participante. Este sistema permite que as DIRs efetuem remanejamento de itens que estejam em excesso, de um município para outro em um período inferior a três meses. O SISPAF teve como conseqüências possibilitar uma meIhor análise e planejamento dos padrões de consumo e uma economia na distribuição dos medicamentos para cada município, possibilitando a expansão do PAF para municípios com população de até 120.000 habitantes.

A previsão inicial de gasto com os medicamentos dos PAF foi de R\$1.200.000/ mês (U\$ $1.050 .000 /$ mês), para atender a uma população de 5 milhões de pessoas. O custo aproximado dos medicamentos do programa é de $\mathrm{R} \$ 2.500 .000 /$ mês (U\$2.200.000/ mês) para atender cerca de 13 milhões de pessoas, a um custo individual de $R \$ 0,19 / \mathrm{hab} /$ mês (U $\$ 0,16$ / hab/ mês).

A distribuição dos medicamentos do PAF é feita pela FURP, baseada na análise do consumo, elaborada pelo SISPAF, consoante cronograma previamente estabelecido. Os municípios consolidam relatórios de consumo mensal de suas UBSs, que são remetidos às DIRs. Estas, por sua vez, consolidam os mapas de consumo de suas regiões, enviando a informação consolidada para a Secretaria Estadual de Saúde que faz os pedidos à FURP de acordo com o crograma de pedido/entrega. O prazo estabelecido entre pedido e entrega é de aproximadamente sete dias. A entrega dos medicamentos é feita com regularidade diretamente às UBSs sempre pelo mesmo funcionário da FURP e é recebida por pessoas autorizadas pelo Secretário Municipal de Saúde. A periodicidade da entrega é trimestral em dias fixos, seguindo um cronograma preestabelecido.

O acompanhamento e a supervisão do PAF em cada um dos municípios, desde a sua implantação, é feito pelas DIRs em conjunto com os gestores municipais do programa. As DIRs realizam reuniões locais segundo a necessidade de cada UBS, além de visitas periódicas e regulares aos municípios, avaliando in loco como está o nível de consumo dos 40 itens do PAF. 
Visando incentivar a prescrição racional e as boas práticas de estocagem e dispensação de medicamentos, foram elaborados materiais informativos escritos e audiovisuais. É distribuído ainda material informativo para os usuários e promovidos seminários de sensibilização para médicos, farmacêuticos, enfermeiros e secretários de saúde.

Até junho de 1996, segundo o Departamento de Planejamento e Avaliação da Coordenadoria de Saúde do Interior (DPA/CSI/SESSP, 1996), entre os resultados alcançados pelo PAF destacam-se: a efetiva relação de co-parceria entre o estado e os municípios; utilização dos medicamentos do programa como instrumento para reorganização dos serviços; recuperação da credibilidade e viabilidade de planejamento da linha de produção da FURP e a integração da assistência farmacêutica às ações e programas de saúde priorizados pelo município.

\section{Estado de Minas Gerais: Farmácia Essencial}

M inas Gerais foi o terceiro estado brasileiro a implementar uma política de medicamentos para o nível primário de saúde pelo Projeto Farmácia Essencial (PFE) e já contou com a experiência neste tipo de programa dos estados de São Paulo e Paraná. O PFE dispõe de uma relação de 36 medicamentos, sendo 29 princípios ativos, que atendem às patologias mais freqüentes do estado. Utilizou-se como critério para a seleção dos medicamentos estudos de demanda feitos pelas Diretorias Regionais de Saúde (DRSs), da lista de medicamentos da Farmácia Básica CEME de 1987, da Oitava Lista de Medicamentos Essenciais da OMS, da Lista de Medicamentos dos programas de São Paulo e Paraná, com base nas quais foi feita uma ponderação de acordo com a linha de produção da Fundação Ezequiel Dias (FUNED) e os recursos disponíveis, considerando também o critério de prioridade para o tratamento da maioria das patologias atendidas pelas UBSs. A quantificação dos medicamentos foi feita para suprir as necessidades de um grupo de 3.000 habitantes por um período de três meses (FUNED, 1997; SESM G/ FU NED, 1997).

A contrapartida dos municípios ao recebimento dos medicamentos são: assinatura de convênio com a Secretaria Estadual de Saúde/ Fundação Ezequiel Dias (SES/ FUNED); complementar as necessidades de medicamentos para o atendimento da atenção ambulatorial; utilizar os medicamentos exclusivamente na rede do SUS, só podendo ser fornecidos mediante prescrição da unidade de saúde que recebe a
FE; organizar seu armazenamento, distribuição e dispensação; criar condições físicas e operacionais para a organização da assistência farmacêutica; apresentar mapa de consumo trimestral da FE devidamente preenchido e assinado pelo responsável; oferecer recursos humanos com perfil adequado à assistência farmacêutica, promovendo sua capacitação; padronizar condutas terapêuticas para o tratamento das patologias predominantes, implantando protocolo de tratamento; assegurar investimentos e financiamentos crescentes para o setor saúde, informando as aplicações financeiras feitas no setor; organizar a assistência à saúde, estruturando sua rede dentro do preconizado pelo SUS; utilizar instrumento legal para formalizar os serviços de assistência farmacêutica dentro da estrutura de assistência à saúde; implementar sistema de controle e avaliação sobre a utilização de medicamentos; criar instrumentos que colaborem no processo de informação e conscientização da população sobre a utilização de medicamentos. O não cumprimento das obrigações pelo município implicará suspensão provisória do fornecimento de medicamentos ou no cancelamento do convênio. O convênio prevê ainda contrapartidas da SES/FUNED que são: garantir a distribuição de cotas trimestrais; repassar aos municípios informações técnicas sobre medicamentos e assessorá-los na organização da assistência farmacêutica; avaliar periodicamente a execução do programa bem como revisar a relação de medicamentos.

Como São Paulo, o estado de Minas Gerais também possui um Sistema de Informação do Programa Farmácia Essencial - SISAFE - que apresenta os mesmos objetivos e estrutura do SISPAF. No estado de Minas Gerais, entretanto, o SISAFE encontra-se totalmente informatizado, inclusive no nível local.

O PFE, que destina-se a municípios com população até 20.000 habitantes, teve início em maio de 1997 cuja implantação ocorre em três etapas. Na primeira etapa, de agosto a dezembro de 1997, o programa foi implantado nos 250 municípios mais carentes do estado com população até 20.000 habitantes, selecionados com base no Índice de Desenvolvimento Humano (IDH), da Fundação João Pinheiro. No segundo ano, a partir de janeiro de 1998 previa-se a implantação em mais 300 municípios utilizando os mesmos critérios e, no terceiro ano (1999), em mais 155 municípios, totalizando 705 municípios com população inferior a 20.000 habitantes, atendendo a uma população total de 6.000 .000 de pessoas. Em fevereiro de 1998, o PFE encontra-se implantado 
em 382 municípios, atendendo a uma população de 3 milhões de habitantes, que corresponde então a cerca de $18 \%$ da população total do estado.

O IDH (IPEA,1996) é composto por três componentes básicos do desenvolvimento humano: longevidade, conhecimento (nível educacional) e padrão de vida. Valendo-se destes componentes é feito o cálculo do IDH, pontuando os municípios conforme o seu grau de desenvolvimento. Este índice foi utilizado também para estabelecer a ordem de inclusão dos municípios no PFE, ou seja, os municípios com menor IDH foram incluídos primeiro no programa. A estratégia de implantação do PFE inicia-se na sensibilização dos municípios para adesão ao programa e capacitação dos profissionais necessários à sua implantação, por intermédio de seminários regionais, incluindo ainda internato rural de acadêmicos de farmácia, contrapartida dos municípios e controle de estoque para a reposição dos medicamentos. Esses seminários contam com a presença obrigatória do Secretário Municipal de Saúde, médicos, farmacêuticos e auxiliares de saúde e é uma condição estabelecida para o recebimento dos medicamentos.

O gasto mensal com medicamentos do PFE é de cerca de $\mathrm{R} \$ 517.000$ (U\$451.000) que equivale a um custo individual de aproximadamente $R \$ 0,17 /$ hab/ mês (U \$0,15/hab/ mês).

O programa é gerenciado pelos gestores municipais do SUS, com apoio técnico das DRSs e supervisionado pela FUNED.

A distribuição dos medicamentos é feita trimestralmente pela FUNED em parceria com a SES/ DRS, diretamente às UBSs, em caminhões próprios, sendo os motoristas treinados para a entrega. No momento do recebimento, os medicamentos são conferidos em relação às suas quantidades e condições de apresentação (inspeção física e controle da validade) e assinado um recibo pelo responsável pelo recebimento no município. As quantidades estabelecidas para o módulo-padrão funcionam como um estoque mínimo, podendo ser alteradas de acordo com a necessidade de cada município. Mensalmente os municípios enviam às DRSs Mapa de Movimentação de Medicamentos, que são consolidados trimestralmente por estas e enviados à FUNED para reposição dos estoques. Desta forma, a reposição dos medicamentos é feita em função da quantidade consumida durante um período de três meses.

Estão previstas no PFE supervisões técnicoadministrativas periódicas a serem realizadas pelas DRSs e avaliações do programa feitas pela DRS/SES/ FUNED que terão por base as in- formações sobre consumo médio das unidades obtidas no SISAFE.

Visando incentivar as boas práticas de estocagem e dispensação de medicamentos, são elaborados materiais informativos escritos com orientações sobre o preenchimento correto dos formulários, além de informações técnicas. São ainda promovidos seminários de sensibilização para médicos, farmacêuticos, enfermeiros e secretários de saúde.

Na Tabela 1 está apresentado um resumo dos principais componentes dos programas analisados com o objetivo de facilitar sua comparação.

Elenco de medicamentos, seleção dos municípios e logística de distribuição: três ações com vistas a assegurar o acesso

O primeiro passo para um sistema de fornecimento de medicamentos eficiente e eficaz é a seleção adequada de medicamentos. A sel eção tem por objetivo limitar o número de medicamentos a serem adquiridos para os diversos níveis de atenção, conduzindo às seguintes conseqüências: melhor suprimento; promove a prescrição mais racional, pois facilita a divulgação de informações sobre os medicamentos; facilita os esforços para promover a educação do paciente e o uso correto de medicamentos, por concentrarem-se em um número limitado destes; reduz o gasto com medicamentos, tornando-os acessíveis às populações cujas necessidades básicas de saúde não são atendidas. A seleção de medicamentos tem um impacto considerável sobre a qualidade da atenção à saúde e o custo do tratamento, sendo, por isso, uma das áreas onde a intervenção é mais custo-efetiva (MSH, 1997; WHO, 1997).

O processo de seleção de medicamentos essenciais começa com a definição de uma lista de doenças mais prevalentes para cada nível do sistema de saúde, identificando tratamentos de primeira escolha, selecionando os medicamentos e suas apresentações e decidindo que medicamento deve estar disponível em cada nível de atenção. O tratamento de primeira escolha para cada problema de saúde é a base para a lista de medicamentos essenciais, para o formulário nacional e as normas de tratamento (MSH, 1997; WHO, 1997).

A seleção dos medicamentos para os programas nos três estados seguem critérios diferentes. Entretanto, nenhum dos estados estudados utilizou o perfil de morbidade como ponto de partida para a seleção dos medicamentos, como recomendado pela OMS. 
Tabela 1

Resumo esquemático dos principais componentes dos programas de medicamentos dos Estados de Paraná, São Paulo e Minas Gerais.

\begin{tabular}{|c|c|c|c|}
\hline Componente do programa & Paraná & São Paulo & Minas Gerais \\
\hline Seleção dos medicamentos & $\begin{array}{l}\text { Critérios de inclusão: constar } \\
\text { da RENAME; serem eficazes, } \\
\text { seguros e disponíveis para } \\
\text { aquisição ao menor custo } \\
\text { possível. } \\
\text { Número de itens: } 32 \text { princípios } \\
\text { ativos em } 40 \text { apresentações }\end{array}$ & $\begin{array}{l}\text { Critérios de inclusão: demanda } \\
\text { histórica de consumo de } \\
\text { medicamentos, fornecida pelos } \\
\text { municípios; Sétima Lista de } \\
\text { Medicamentos Essenciais da } \\
\text { OMS; capacidade de produção } \\
\text { do medicamento pela FURP } \\
\text { (Fundação para o Remédio } \\
\text { Popular). } \\
\text { Número de itens: } 32 \text { princípios } \\
\text { ativos em } 40 \text { apresentações. }\end{array}$ & $\begin{array}{l}\text { Critérios de inclusão: estudo de } \\
\text { demanda feito pelas Diretorias } \\
\text { Regionais de Saúde (DRSs), lista } \\
\text { de medicamentos da Farmácia } \\
\text { Básica CEME de 1987, O itava } \\
\text { Lista de Medicamentos } \\
\text { Essenciais da O MS, Lista de } \\
\text { Medicamentos dos programas } \\
\text { de São Paulo e Paraná, linha de } \\
\text { produção da Fundação Ezequiel } \\
\text { Dias (FUNED), patologias mais } \\
\text { freqüentes atendidas pelas } \\
\text { UBSs. } \\
\text { Número de itens: } 29 \text { princípios } \\
\text { ativos em } 36 \text { apresentações. }\end{array}$ \\
\hline Seleção dos municípios & Índice de Salubridade & Populacional & $\begin{array}{l}\text { Populacional e Índice de } \\
\text { Desenvolvimento Humano. }\end{array}$ \\
\hline Aquisição & $\begin{array}{l}\text { CEME, produção no CEMEPAR, } \\
\text { em laboratórios públicos esta- } \\
\text { duais conveniados, laboratórios } \\
\text { oficiais de outros estados ou de } \\
\text { laboratórios privados. }\end{array}$ & FURP & $\begin{array}{l}\text { FUNED e laboratórios oficiais } \\
\text { conveniados }\end{array}$ \\
\hline Distribuição & $\begin{array}{l}\text { Centralizado: CEMEPAR } \rightarrow \\
\text { Regionais de Saúde } \rightarrow \text { Secre- } \\
\text { tarias M unicipais de Saúde } \\
\rightarrow \text { UBS. }\end{array}$ & Descentralizada: FURP $\rightarrow$ UBS & Descentralizada: FUNED $\rightarrow$ UBS \\
\hline $\begin{array}{l}\text { Reposição de } \\
\text { medicamentos }\end{array}$ & $\begin{array}{l}\text { Módulo-padrão completo, } \\
\text { trimestralmente }\end{array}$ & $\begin{array}{l}\text { De acordo com o consumo, } \\
\text { trimestralmente }\end{array}$ & $\begin{array}{l}\text { De acordo com o consumo, } \\
\text { trimestralmente }\end{array}$ \\
\hline Controle de qualidade & Laboratórios credenciados & FURP & FUNED \\
\hline $\begin{array}{l}\text { Controle de estoque } \\
\text { informatizado }\end{array}$ & Nível central & $\begin{array}{l}\text { Nível central, regional e local } \\
\text { (alguns municípios) }\end{array}$ & Nível central, regional e local \\
\hline Seminário de implantação & Não & Sim & Sim \\
\hline $\begin{array}{l}\text { Custo mensal com } \\
\text { medicamentos }\end{array}$ & $\begin{array}{l}\mathrm{R} \$ 912.000 \\
(\mathrm{R} \$ 0,23 / \text { hab/mês })\end{array}$ & $\begin{array}{l}\mathrm{R} \$ 2.500 .000 \\
(\mathrm{R} \$ 0,19 / \text { hab } / \text { mês })\end{array}$ & $\begin{array}{l}\mathrm{R} \$ 517.000 \\
(\mathrm{R} \$ 0,17 / \text { hab/mês) }\end{array}$ \\
\hline $\begin{array}{l}\text { Porcentagem da } \\
\text { população atendida1 }\end{array}$ & $43 \%$ do estado & $45 \%$ do estado ( $74 \%$ da CSI) & $18 \%$ do estado \\
\hline
\end{tabular}

RENAME = Relação Nacional de Medicamentos Essenciais; OMS = O rganização Mundial da Saúde; CEME = Central de Medicamentos; CEMEPAR = Centro de Medicamentos do Paraná; UBS = Unidade Básica de Saúde; CSI = Coordenadoria de Saúde do Interior.

1 Porcentagem da população atendida até o momento em que foi realizado este estudo.

Os Estados de São Paulo e Minas Gerais apresentam a vantagem de possuírem laboratórios farmacêuticos, com capacidade de distribuição de medicamentos, fazendo parte da estrutura das SESs. Isto garante agilidade administrativa e eficiência na distribuição, entre outras vantagens, justificando-se assim, o fato destes estados utilizarem como um critério importante para a seleção dos medicamentos, as linhas de produção da FURP e FUNED. O Estado do Paraná, entretanto, seguiu basica- mente o modelo CEME para a Farmácia Básica de 1987.

Os Estados de São Paulo e Minas Gerais produzem por intermédio da FURP e da FUNED, respectivamente, todos os medicamentos necessários aos seus programas. Já o CEMEPAR, no Estado do Paraná, produz apenas uma parte dos medicamentos do PFB-PR, sendo o restante adquirido de laboratórios oficiais e indústrias privadas, o que torna este programa o mais caro entre os dos três estados (Tabela 1). 
A última revisão da RENAME (MS, 1998) preconizou como um dos critérios para a seleção dos medicamentos, a redução da variabilidade de apresentações, com a finalidade de facilitar os procedimentos logísticos, selecionando as que proporcionam maior flexibilidade posológica. A utilização deste critério propiciou uma extensa discussão sobre a apresentação a ser selecionada, tendo em vista estudos recentes que mostram a eficácia terapêutica de alguns medicamentos, como por exemplo a hidroclorotiazida, em doses inferiores às utilizadas até o momento. Tomando por base esta discussão, comparando-se as listas de medicamentos dos três estados em relação ao princípio ativo e sua forma de apresentação, com as RENAMEs 83 e 98 (MS, 1989, 1998) obtiveramse os resultados apresentados na Tabela 2.

A análise da Tabela 2 mostra uma grande adesão dos três estados à RENAME/ 83, que era a versão disponível à época da elaboração das listas de medicamentos dos programas estudados. Contudo, quando se comparam as relações dos programas com a RENAM E/ 98, observa-se uma diminuição na adesão dos três estados a esta lista, indicando a necessidade de revisão e atualização das relações de medicamentos dos três programas.

Os critérios utilizados para a sel eção dos municípios que receberiam os medicamentos dos programas foram, igualmente, diferentes nos três estados. Os Estados do Paraná e Minas Gerais utilizaram um método lógico para classificar os municípios que receberiam seus programas que foram o IS e o IDH, respectivamente. $\mathrm{O}$ uso destes índices possibilita um melhor gerenciamento dos recursos financei ros e uma seleção mais criteriosa dos municípios, sendo uma alternativa para os estados que não possuem recursos financeiros para o atendimento de toda a população. O Estado de São Paulo, todavia, utilizou como critério para a seleção dos municípios o tamanho da sua população.

Tabela 2

Índices de adesão por estados e por listas de medicamentos, com relação aos princípios ativos e suas apresentações.

\begin{tabular}{lccc}
\hline Estado & RENAME/83 & RENAME/98 & $\begin{array}{l}\text { Total de PA } \\
\text { da lista básica }\end{array}$ \\
\hline Paraná & $40(100 \%)$ & $29(72,5 \%)$ & $40(100 \%)$ \\
São Paulo & $37(92,5 \%)$ & $26(65,0 \%)$ & $40(100 \%)$ \\
Minas Gerais & $35(97,2 \%)$ & $26(72,2 \%)$ & $36(100 \%)$ \\
\hline
\end{tabular}

RENAME = Relação Nacional de Medicamentos Essenciais; PA = Princípio Ativo.
Quanto ao sistema de distribuição de medicamentos utilizado, o Estado do Paraná adotou o modelo centralizado, como o utilizado pela extinta CEME. Este modelo apresenta como vantagens o fato de ser fácil de monitorar e de permitir o controle, por parte do governo, de todo o sistema. Como desvantagens, destacam-se o alto custo de manutenção dos almoxarifados em termos de equipamentos e pessoal, transporte e outros custos operacionais, além de estar sujeito a interferências políticas, entre outras (MSH, 1997) e apresentar maior possibilidade de desvios e perda por validade. O fato de a distribuição ser centralizada e de haver informatização apenas no nível central, dificulta o planejamento de reposição baseada em consumo. Talvez esta seja uma das razões para a reposição ocorrer por módulo-padrão no Estado do Paraná.

Os Estados de São Paulo e Minas Gerais adotaram o modelo de distribuição descentralizado que apresenta como vantagens o fato de eliminar os custos de manutenção de estoques e distribuição por parte do governo; manter os benefícios de preços baixos obtidos com o sistema centralizado de aquisição e reduzir os custos com inventário e perda de validade de medicamentos. Como desvantagem, ressaltamos a exigência de coordenação e monitoramento das entregas (MSH,1997). O MSH (1997) destaca ainda como desvantagem inerente ao sistema descentralizado o fato de nem todos os fornecedores serem capazes de assegurar a distribuição (pode reduzir a competição e el evar os custos); além da distribuição direta por múltiplos fornecedores (especialmente para áreas distantes) ser ineficiente, podendo aumentar os custos. Entendemos, no entanto, que estas desvantagens não se aplicam aos casos analisados.

Quando se discutem sistemas de distribuição para programas estaduais de medicamentos, deve-se considerar a realidade de cada estado em relação às variáveis que interferem nos diferentes modelos, para que se possa achar o que seja mais adequado. Mas, sempre que possível de ser adotado, o modelo descentralizado com um bom sistema de informação é mais eficiente e econômico, principalmente quando o(s) laboratório(s) produtor(es) encontram-se sediado(s) no próprio estado. A exemplo do Estado de São Paulo, a economia gerada com um sistema de distribuição descentralizado e um sistema de informação eficaz, resultou em uma economia que possibilitou a expansão do PAF para outros municípios.

É interessante destacar a estratégia utilizada pelos Estados de São Paulo e Minas Gerais 
para esclarecer e comprometer os gestores municipais e representantes das Diretorias Regionais de Saúde, acerca da importância dos seus programas, através de seminários de implantação e sensi bilização. Nestes seminários adotase uma metodologia participativa, que mescla técnicas pedagógicas e ludo-pedagógicas, com resultados mais efetivos no que se refere ao entendimento da proposta do programa. Estes seminários promovem ainda a integração entre os profissionais da área da saúde de diversos municípios, motivando-os para o trabalho com os programas; permitem uma análise e avaliação dos programas por parte dos profissionais, bem como discussões sobre sua viabilização e operacionalização, além de esclarecer as dúvidas existentes.

\section{Considerações finais}

Vivemos em 1998 a implementação de um programa de âmbito nacional (PFB) que envolve, direta e indiretamente, 4.175 municípios, atingindo uma população estimada em 35 milhões de habitantes, que corresponde a cerca de $22 \%$ da população do país. Iniciando com uma primeira fase de distribuição centralizada pela esfera federal do SUS, pretende-se uma descentralização do programa, mediante o estímulo para que as unidades da federação, progressivamente, criem seus próprios programas estaduais de assistência farmacêutica (MS, 1997a, 1997b). Essa proposta vem ao encontro da reorientação da assistência farmacêutica adotada na Política Nacional de Medicamentos (MS, 1999).

Os Estados do Paraná, São Paulo e Minas Gerais implantaram os modelos descentralizados de assistência farmacêutica, quando ainda existia uma cultura e prática centralizadora ditada pelo Sistema CEME, sendo portanto uma iniciativa pioneira e voltada para a proposta atual de política de medicamentos do país.

Observa-se, como um dos méritos nas iniciativas dos três estados, o fato de adotarem uma metodologia administrativa moderna e participativa. Destacando-se a participação dos níveis locais de atendimento, representados pelos municípios, nas discussões dos programas, envolvendo-os e responsabilizando-os pela contrapartida ao recebimento dos medicamentos.

A assistência farmacêutica deve ser abordada como um dos componentes da promoção integral à saúde que pode utilizar o medicamento como um importante instrumento para o aumento da resolubilidade do atendimento ao paciente. As experiências dos três estados, aqui analisadas, mostram que isto é possível.

\section{Referências}

ABBAS, R. \& BERMUDEZ, J., 1993. Preços no Brasil superam em muito os do mercado internacional. Tema, ano XI, outubro:4-5.

BERM UDEZ, J., 1992. Remédios: Saúde ou Indústria? A Produção de Medicamentos no Brasil. Rio de Janeiro: Editora Relume Dumará.

BERMUDEZ, J., 1994. Medicamentos genéricos: Uma alternativa para o mercado brasileiro. Cadernos deSaúdePública, 10:268-278.

BERMUDEZ, J. A. Z. \& POSSAS, C. A., 1995. Análisis crítico de la política de medicamentos en el Brasil. Boletín de la Oficina Sanitaria Panamericana, 119:270-277.

BERMUDEZ, J., 1995. Indústria Farmacêutica, Estado eSociedade: Crítica da Política de Medicamentos no Brasil. São Paulo: Editora Hucitec/ Sociedade Brasileira de Vigilância de Medicamentos SOBRAVIME.

BERMUDEZ, J.; ROZENFELD, S. \& PORTELA, M. C., 1997. Avaliação do Programa Farmácia Básica Brasil, 1997/1998. Projeto submetido ao Ministério da Saúde. Rio de Janeiro: Escola Nacional de Saúde Pública/ Fundação Oswaldo Cruz. (mimeo.)
CASTELO, A.; COLOMBO, A. L. \& HOLBROOK, A. M., 1991. Production and marketing of drugs in Brazil. Journal of Clinical Epidemiology, 44(Sup.2):21S$28 \mathrm{~S}$.

CSI/ SESSP (Coordenadoria de Saúde do Interior/ Secretaria de Estado de Saúde de São Paulo), 1996. Sumário do Projeto de Expansão do Programa Estadual de Assistência Farmacêutica no Estado de São Paulo. São Paulo: SESSP.

CSI/ SESSP (Coordenadoria de Saúde do Interior/Secretaria de Estado de Saúde de São Paulo), 1998. Programa Estadual de Assistência Farmacêutica no Estado de São Paulo. São Paulo: SESSP.

DPA/CSI/ SESSP (Departamento de Planejamento e Avaliação/Coordenadoria de Saúde do Interior/ Secretaria de Estado de Saúde de São Paulo), 1996. Programa Estadual de Assistência Farmacêutica. São Paulo: SESSP.

FUNED (Fundação Ezequiel Dias), 1997. Relatório da Implantação do Projeto Farmácia Essencial. Minas Gerais: FUNED.

FURP (Fundação para o Remédio Popular), s/d. Dose Certa: O Programa deAssistência Farmacêutica do Estado de São Paulo. São Paulo: FURP. 
GAF/SESPR (Grupo de Assistência Farmacêutica/ Secretaria Estadual de Saúde do Paraná), 1995. Paraná Mais Saúde. Curitiba: Centro de Medicamentos do Paraná.

GTPA/CSI/SESSP (Grupo Técnico de Planejamento e Avaliação/Coordenadoria de Saúde do Interior/ Secretaria de Estado de Saúde de São Paulo), 1996. Programa Estadual de Assistência Farmacêutica. São Paulo: SESSP.

IPEA (Instituto de Pesquisas Econômicas Aplicadas), 1996. Relatório sobre o Desenvol vimento Humano no Brasil. Rio de Janeiro: IPEA/Brasília: PNUD.

MEDICI, A. C.; OLIVEIRA, F. \& BELTRÃO, K. I., 1991. A Política de Medicamentos no Brasil. Rio de Janeiro: Escola Nacional de Ciências Estatísticas/Instituto Brasileiro de Geografia e Estatística.

MS (Ministério da Saúde), 1989. Memento Terapêutico CEME da Relação Nacional de Medicamentos Essenciais - RENAME. Brasília: MS.

MS (Ministério da Saúde), 1997a. Programa Farmácia Básica. Brasília: MS.

MS (Ministério da Saúde), 1997b. Programa Farmácia Básica. Brasília: MS. Disponível na internet na home page \tttp:// www.saude.gov.br/farmacia. htm>.

MS (Ministério da Saúde), 1997c. Memento Terapêutico Farmácia Básica: Relação de Medicamentos. Brasília: MS.

MS (Ministério da Saúde), 1998. Relação Nacional de Medicamentos Essenciais (RENAME). Brasília: MS. Disponível na internet na home page বhttp:// www.saude.gov.br/ rename.htm>.
MS (Ministério da Saúde), 1999. Política Nacional de Medicamentos. Brasília: Secretaria de Políticas de Saúde, Ministério da Saúde.

MSH (Management Sciences for Health), 1997. Managing Drug Supply: The Sel ection, Procurement, Distribution, and Use of Pharmaceuticals. USA: Kumarian Press.

PEPE, V. L. E. \& VERAS, C. M.T., 1995. A Prescrição Mé dica. Rio de Janeiro: Instituto de Medicina Social/ Universidade do Estado do Rio de Janeiro.

PEREIRA, A. A., 1995. Produção Descentralizada de Medicamentos Essenciais no Instituto de Tecnologia em Fármacos do Estado do Rio de Janeiro: Um Diagnóstico Estratégico de sua Implementação. Dissertação de Mestrado, Rio de Janeiro: Escola Brasileira de Administração Pública, Fundação Getúlio Vargas.

SESM G/FUNED (Secretaria do Estado da Saúde de Minas Gerais/Fundação Ezequiel Dias), 1997. Projeto Farmácia Essencial: "Cidadania e Saúde". Minas Gerais: FUNED.

SINDUSFARM (Sindicato da Indústria de Produtos Farmacêuticos no Estado de São Paulo), 1998. Resultados do ano de 1997. Boletim SINDUSFARM, $1: 1-2$.

STROZZI, J. B., 1997. SUS Pense: Uma Reflexão Epidemiológica sobre o SUS e outras Aplicações Acadêmicas. Londrina: CEBES.

WHO (World Health Organization), 1988. TheWorld Drug Situation. Geneva: WHO.

WHO (World Health Organization), 1997. The Use of Essential Drugs. WHO Technical Report Series 867. Geneva: WHO. 